\title{
A novel gene mutation in Berardinelli Seip Syndrome: Three case reports
}

\author{
Gungor $\mathrm{A}^{1}$, Gokkaya $\mathrm{N}^{1}$, Karadeniz $\mathrm{Y}^{1}$, Bilen $\mathrm{A}^{2}$, Bilen $\mathrm{H}^{1}$
}

${ }^{1}$ Atatürk University Faculty of Medicine, Internal Medicine, Endocrinology and Metabolism Department, Erzurum, Turkey

${ }^{2}$ Atatürk University Faculty of Medicine, Internal Medicine, Erzurum, Turkey

\section{Introduction:}

Case Report:

\section{Discussion:}

Although BSCL patients are born with typical phenotypic features, complications settle with the progression of the disease. A serious disease, BSCL should be followed and treated by multidisciplinary due to multiorgan impairments. In addition to symptomatically treatment of BSCL complications, leptin, which is a cytokine contributing to disease pathogenesis, replacement is becoming more important recently.

Berardinelli Seip Congenital Lypodystrophy(BSCL) is a rare otosomal recessive disease characterized by major criteria which are lypoatrophy, acromegaloid features, hepatomegaly, hypertriglyceridemia, insulin resistance and minor citeria which are hypertrophic cardiomyopathy, psycomotor retardation, hirsutism, precocious puberty, long bone cysts, phlebomegaly. The presence of three major criteria or two major plus two minor criteria required for diagnosis of BSCL. We report three BSCL cases who are all from the same family and the same genetic mutation of BSCL 2 homozygote pQ94X (stop codon) which is encountered the first time in literature.

The first case, 25 years old woman had appearance of acromegaloid face, hyperpigmentation on her skin, widespread acanthosis nigricans, diabetes mellitus, hepatosplenomegaly, prominent decrease of subcutaneous adipose tissue, hypertrophied limb and shoulder muscles. She had also chronic renal insufficiency requiring hemodialysis, cirrhosis of liver, pericardial effusion and hypertropy of left ventricul and interventricular septum. The second case is 20 years old man, the brother of the first one. He had acromegaloid face, darkening skin, acanthosis nigricans, hepatosteatosis, hepatomegaly, diabetes mellitus and hypertriglyceridemia. The third case is their cousin who is 16 years old man. He had similar face characteristic with them, hyperpigmented skin, acanthosis nigricans, hepatosteatosis, hepatomegaly, diabetes mellitus and absence of subcutaneous adipose tissue on entire body MRI. Genetic analysis of three patients showed BSCL 2 homozygote PQ94X(stop codon) mutation which is encountered the first time in literature. 